\title{
DYNAMICS OF REACTION-DIFFUSION EQUATIONS WITH NONLOCAL BOUNDARY CONDITIONS
}

\author{
By \\ C. V. PAO \\ Department of Mathematics, North Carolina State University, Raleigh, North Carolina
}

\begin{abstract}
The purpose of this paper is to investigate the existence, uniqueness, and dynamics of a nonlinear reaction-diffusion equation with a nonlocal boundary condition which is motivated by a model problem arising from quasi-static thermoelasticity. The method of upper and lower solutions is used to obtain some existence-comparison results for both the time-dependent problem and its corresponding steady-state problem. A sufficient condition for the uniqueness of a steadystate solution is given. The comparison and uniqueness results are used to show the dynamical behavior of time-dependent solutions as well as their monotone convergence to a steady-state solution. Also given is a necessary and sufficient condition for the convergence of time-dependent solutions in relation to a steady-state solution which is not explicitly known. These results lead to the global stability of a steady-state solution for some special cases, including the model problem from thermoelasticity.
\end{abstract}

1. Introduction. In recent years considerable attention has been given to reactiondiffusion systems where nonlocal reaction functions are involved either in the differential equations or on the boundary conditions (cf. [1-11]). One of these systems arises from quasi-static thermoelasticity where the entropy is governed by a parabolic equation under a nonlocal boundary condition. A linear mathematical model in slab geometry was derived by Day $[3,4]$ where some decay property of the time-dependent solution was investigated. The same decay problem was extended by Friedman [7] to a more general parabolic equation in a multi-dimensional domain. Using the method of upper and lower solutions, Deng [6] obtained a comparison result and a decay property of the solution for a nonlinear reaction-diffusion equation, including the existence of a local solution. The equations considered in all of the above work

Received October 26, 1992.

1991 Mathematics Subject Classification. Primary 35K57, 35B40, 35K20.

(C)1995 Brown University 
may be expressed in the form

$$
\begin{array}{ll}
u_{t}-L u=f(x, u) & (t>0, x \in \Omega), \\
B[u]=\int_{\Omega} K(x, y) u(t, y) d y & (t>0, x \in \partial \Omega), \\
u(0, x)=u_{0}(x) & (x \in \Omega),
\end{array}
$$

where $\Omega$ is a bounded smooth domain in $\mathbb{R}^{n}(n=1,2, \ldots), \partial \Omega$ is the boundary of $\Omega$, and $L$ is a uniformly elliptic operator given in the form

$$
L u \equiv \sum_{i, j=1}^{n} a_{i j}(x) u_{x_{i} x_{j}}+\sum_{j=1}^{n} b_{j}(x) u_{j}
$$

The uniform ellipticity of $L$ is in the sense that the coefficient matrix $\left(a_{i j}(x)\right)$ is positive definite for all $x \in \bar{\Omega}$. The boundary operator $B[\cdot]$ is given by

$$
B[u] \equiv \alpha_{0} \partial u / \partial \nu+u,
$$

where $\alpha_{0} \geq 0$ is a constant and $\partial u / \partial \nu$ is the outward normal derivative of $u$ on $\partial \Omega$. Problem (1.1) is reduced to the problem considered in [3, 4] and [7] when $\alpha_{0}=0$ and $f(x, u)=c(x) u$ (with $c(x) \leq 0$ ), and to the one in [6] when $\alpha_{0}=0$ and $L u=\nabla^{2} u$. In this paper, we consider a nonnegative constant $\alpha_{0}$ and a general reaction function $f(x, u)$ where $f(x, 0)$ is not necessarily identically zero. The consideration of $\alpha_{0} \geq 0$ includes the boundary condition of Dirichlet type $\left(\alpha_{0}=0\right)$ and of Robin type $\left(\alpha_{0}>0\right)$, while the case $f(x, 0) \not \equiv 0$ excludes zero as a steadystate solution. We assume that the coefficients of $L$, the kernel $K(x, y)$, and the initial function $u_{0}$ are all smooth functions of their arguments, and $u_{0}$ satisfies the boundary condition in (1.1) at $t=0$. The function $f(x, u)$ is assumed Hölder continuous in $x$ and continuously differentiable in $u$. The above smoothness hypotheses are used to ensure the existence of a classical solution to the time-dependent problem (1.1) as well as to the corresponding steady-state problem which is given by

$$
\begin{array}{rlrl}
-L u & =f(x, u) & & (x \in \Omega), \\
B[u]=\int_{\Omega} K(x, y) u(y) d y & & (x \in \partial \Omega) .
\end{array}
$$

The purpose of this paper is to establish (i) some existence-comparison results for the time-dependent problem (1.1), (ii) existence and uniqueness results for the steadystate problem (1.2), and (iii) the dynamics of problem (1.1) in relation to problem (1.2). Of special concern is the monotone convergence of the time-dependent solution and the estimate of the stability region of a steady-state solution, including the global stability of a solution. Our basic approach to the above problems is the method of upper and lower solutions. To investigate the dynamics of problem (1.1) it is important to know whether a solution to problem (1.2) exists and whether it is unique. Since $f(x, 0)$ is not necessarily assumed identically zero, the existence of a solution to (1.2) is not always guaranteed; and even if such a solution exists, it is, in general, nonuniform in $\bar{\Omega}$. The determination of the dynamics of (1.1) with respect to a nonconstant steady-state solution is much more delicate than that to the zero solution when $f(x, 0) \equiv 0$. 
The plan of the paper is as follows. In Sec. 2 we give some existence-comparison results for the time-dependent problem (1.1). Section 3 is devoted to the existence of maximal and minimal solutions of problem (1.2) as well as the uniqueness of a solution. The dynamics of problem (1.1) is discussed in Sec. 4. Here the monotone convergence of time-dependent solutions, and a necessary and sufficient condition for the convergence of time-dependent solutions to a steady-state solution are given. These results are used to show the global stability of some model problems, including the entropy problem considered in $[3,4,6,7]$.

2. The time-dependent problem. Let $D_{T}=(0, T] \times \Omega, \bar{D}_{T}=[0, T] \times \bar{\Omega}$, and $S_{T}=(0, T] \times \partial \Omega$, where $\bar{\Omega} \equiv \Omega \cup \partial \Omega$ and $T>0$ is finite. Denote by $C^{m}(\Omega)$ the set of $m$-times continuously differentiable functions in $\Omega$, and by $C^{1,2}\left(D_{T}\right)$ the set of functions that are once continuously differentiable in $t \in(0, T]$ and twice continuously differentiable in $x \in \Omega$. Similar notations are used for $C^{m}(\bar{\Omega}), C^{m}\left(\bar{D}_{T}\right)$, etc. To show the existence of a global solution to (1.1) it suffices to consider the problem in the domain $D_{T}$ for an arbitrary $T>0$. Our approach to the existence problem is based on the method of upper and lower solutions. When the kernel $K(x, y)$ is nonnegative on $\partial \Omega \times \Omega$ we have the following definition.

Definition 2.1. A function $\tilde{u} \in C^{1,2}\left(D_{T}\right) \cap C\left(\bar{D}_{T}\right)$ is called an upper solution of (1.1) if it satisfies

$$
\begin{array}{ll}
\tilde{u}-L \tilde{u} \geq f(x, \tilde{u}) & \text { in } D_{T}, \\
B[\tilde{u}] \geq \int_{\Omega} K(x, y) \tilde{u}(t, y) d y & \text { on } S_{T}, \\
\tilde{u}(0, x) \geq u_{0}(x) & \text { in } \Omega .
\end{array}
$$

Similarly, $\hat{u}$ is called a lower solution if it satisfies all the reversed inequalities in (2.1).

It is clear from the above definition that every (classical) solution of (1.1) is an upper solution as well as a lower solution. A pair of upper and lower solutions $\tilde{u}, \hat{u}$ are said to be ordered if $\tilde{u} \geq \hat{u}$ in $\bar{D}_{T}$. Given a pair of ordered upper and lower solutions $\tilde{u}, \hat{u}$ we define

$$
c(t, x) \equiv \max \left\{-\frac{\partial f}{\partial u}(x, u) ; \hat{u} \leq u \leq \tilde{u}\right\} .
$$

By adding a common term $c u$ on both sides of the differential equation in (1.1) we construct a sequence $\left\{u^{(k)}\right\}$ successively from the linear iteration process:

$$
\begin{array}{ll}
u_{t}^{(k)}-L u^{(k)}+c u^{(k)}=c u^{(k-1)}+f\left(x, u^{(k-1)}\right) & \text { in } D_{T}, \\
B\left[u^{(k)}\right]=\int_{\Omega} K(x, y) u^{(k-1)}(t, y) d y & \text { on } S_{T}, \\
u^{(k)}(0, x)=u_{0}(x) & \text { in } \Omega .
\end{array}
$$

The initial iteration $u^{(0)}$ is either the upper solution $\tilde{u}$ or the lower solution $\hat{u}$. It is clear from the hypotheses in the introduction that the sequence given by $(2.3)$ is well defined. Denote the sequence by $\left\{\bar{u}^{(k)}\right\}$ when $u^{(0)}=\tilde{u}$ and by $\left\{\underline{u}^{(k)}\right\}$ when $u^{(0)}=\hat{u}$. The following theorem gives the monotone convergence of these sequences. 
THEOREM 2.1. Let $K(x, y) \geq 0$ and let $\tilde{u}, \hat{u}$ be a pair of ordered upper and lower solutions of (1.1). Then the sequences $\left\{\bar{u}^{(k)}\right\},\left\{\underline{u}^{(k)}\right\}$ given by $(2.3)$ with $\bar{u}^{(0)}=\tilde{u}$ and $\underline{u}^{(0)}=\hat{u}$ converge monotonically from above and below, respectively, to a unique solution $u \equiv u(t, x)$ of $(1.1)$. Moreover,

$$
\hat{u} \leq \underline{u}^{(k)} \leq \underline{u}^{(k+1)} \leq u \leq \bar{u}^{(k+1)} \leq \bar{u}^{(k)} \leq \tilde{u} \quad \text { in } \bar{D}_{T},
$$

where $k=1,2, \ldots$.

Proof. Since the proof is similar to that for a nonlocal problem in [9], where a more general parabolic system with coupled nonlocal boundary conditions is considered, we only give a sketch as follows: Let $w=\bar{u}^{(0)}-\bar{u}^{(1)}=\tilde{u}-\bar{u}^{(1)}$. By (2.3) and (2.1),

$$
\begin{aligned}
w_{t}-L w+c w & =\left(\tilde{u}_{t}-L \tilde{u}+c \tilde{u}\right)-\left(c \bar{u}^{(0)}+f\left(x, \bar{u}^{(0)}\right)\right) \\
& =\tilde{u}_{t}-L \tilde{u}-f(x, \tilde{u}) \geq 0 \text { in } D_{T}, \\
B[w] & =B[\tilde{u}]-\int_{\Omega} K(x, y) \bar{u}^{(0)}(t, y) d y \geq 0 \quad \text { on } S_{T}, \\
w(0, x) & =\tilde{u}(0, x)-u_{0}(x) \geq 0 \text { in } \Omega .
\end{aligned}
$$

By an application of the maximum principle or the positivity lemma for parabolic operators, $w \geq 0$ in $\bar{D}_{T}$ (cf. $[9,13]$ ). This proves the relation $\bar{u}^{(1)} \leq \bar{u}^{(0)}$. A similar argument gives $\underline{u}^{(1)} \geq \underline{u}^{(0)}$. Let $\bar{w}=\bar{u}^{(1)}-\underline{u}^{(1)}$. Then by (2.3), (2.2), and $\bar{u}^{(0)} \geq \underline{u}^{(0)}$,

$$
\begin{aligned}
& \bar{w}_{t}-L \bar{w}+c \bar{w}=c\left(\bar{u}^{(0)}-\underline{u}^{(0)}\right)+f\left(x, \bar{u}^{(0)}\right)-f\left(x, \underline{u}^{(0)}\right) \geq 0, \\
& B[\bar{w}]=\int_{\Omega} K(x, y)\left[\bar{u}^{(0)}(t, y)-\underline{u}^{(0)}(t, y)\right] d y \geq 0, \\
& \bar{w}(0, x)=u_{0}(x)-u_{0}(x)=0 .
\end{aligned}
$$

It follows again from the maximum principle that $\bar{w} \geq 0$ which yields $\bar{u}^{(1)} \geq \underline{u}^{(1)}$. The above conclusions show that $\underline{u}^{(0)} \leq \underline{u}^{(1)} \leq \bar{u}^{(1)} \leq \bar{u}^{(0)}$. It is easily seen by an induction argument that $\left\{\bar{u}^{(k)}\right\}$ and $\left\{\underline{u}^{(k)}\right\}$ possess the monotone property in (2.4). This monotone property implies that the limits

$$
\lim \bar{u}^{(k)}(t, x)=\bar{u}(t, x) \quad \text { and } \quad \lim \underline{u}^{(k)}(t, x)=\underline{u}(t, x) \quad \text { as } k \rightarrow \infty
$$

exist and $\bar{u} \geq \underline{u}$. A regularity argument as in $[9,14]$ shows that $\bar{u}$ and $\underline{u}$ are solutions of (1.1). Moreover, a ladder argument as in $[9,11]$ shows that $\bar{u}=\underline{u}$ and is the unique solution of (1.1).

It is seen from Theorem 2.1 that the existence of a unique solution to (1.1) is guaranteed if $K(x, y) \geq 0$ and there exist a pair of ordered upper and lower solutions. To ensure the existence of upper and lower solutions it is necessary to impose some additional conditions on $K(x, y)$ and $f(x, u)$. For the function $K(x, y)$ we have the following basic requirement:

$$
K(x, y) \geq 0, \quad \int_{\Omega} K(x, y) d y \leq 1 \quad(x \in \partial \Omega, y \in \Omega) .
$$

Theorem 2.2. Let $K(x, y)$ satisfy $(2.5)$ and let $f(x, u)$ satisfy the condition

$$
f(x, m) \geq 0, \quad f(x, M) \leq 0 \quad(x \in \Omega)
$$


for some constants $m \leq 0, M>0$. Then for any $u_{0}$ with $m \leq u_{0} \leq M$, problem (1.1) has a unique solution $u(t, x)$ and

$$
m \leq u(t, x) \leq M \quad(t>0, x \in \bar{\Omega}) .
$$

The solution $u(t, x)$ is nonnegative if $u_{0} \geq 0$ and (2.6) holds with $m=0$.

Proof. It is easily seen from (2.5) and (2.6) that the constant function $\tilde{u} \equiv M$ satisfies the relation

$$
\begin{aligned}
\tilde{u}_{t}-L \tilde{u} & =0 \geq f(x, M)=f(x, \tilde{u}), \\
B[\tilde{u}] & =M \geq \int_{\Omega} K(x, y) \tilde{u}(t, y) d y .
\end{aligned}
$$

Since $\tilde{u}(0, x)=M \geq u_{0}, \tilde{u}$ is an upper solution. Similarly $\hat{u} \equiv m$ satisfies the relation

$$
\begin{aligned}
\hat{u}_{t}-L \hat{u} & =0 \leq f(x, m)=f(x, \hat{u}), \\
B[\hat{u}] & =m \leq \int_{\Omega} K(x, y) \hat{u}(t, y) d y .
\end{aligned}
$$

In view of $\hat{u}(0, x)=m \leq u_{0}(x), \hat{u}$ is a lower solution. The existence of a unique solution $u$ and the relation (2.7) follow from Theorem 2.1. When $u_{0} \geq 0$ and (2.6) holds with $m=0$ the nonnegative property of $u(t, x)$ follows from (2.7).

Theorem 2.2 yields a bounded nonnegative solution of (1.1) when $u_{0} \geq 0$ and (2.6) holds with $m=0$. In case this condition is replaced by the linear growth condition

$$
f(x, u) \leq a(x) u+b(x) \text { for } u \geq 0 \quad(x \in \Omega),
$$

where $a(x), b(x)$ are any continuous functions with $b(x) \geq 0$, then a nonnegative global solution still exists. This global existence result is contained in the following:

Theorem 2.3. Let $f(x, 0) \geq 0$ and let (2.5) and (2.8) hold with $b(x) \geq 0$. Then for any $u_{0} \geq 0$ there exist constants $\rho, \gamma$ such that a unique global solution $u(t, x)$ to (1.1) exists and satisfies

$$
0 \leq u(t, x) \leq \rho e^{y t} \quad(t>0, x \in \bar{\Omega}) .
$$

Moreover, $u(t, x) \rightarrow 0$ uniformly in $\bar{\Omega}$ as $t \rightarrow \infty$ when $a(x)<0$ and $b(x) \equiv 0$ in $\bar{\Omega}$.

Proof. It is obvious from $f(x, 0) \geq 0$ that $\hat{u}=0$ is a lower solution of (1.1). In view of (2.8), the function $\tilde{u}=\rho e^{\gamma t}$ is a positive upper solution if $\rho \geq u_{0}$ and

$$
\begin{aligned}
\gamma \rho e^{\gamma t} & \geq a(x) \rho e^{\gamma t}+b(x), \\
\rho e^{\gamma t} & \geq \int_{\Omega} K(x, y) \rho e^{\gamma t} d y .
\end{aligned}
$$

By (2.5), the second inequality is trivially satisfied while the first inequality is equivalent to

$$
\gamma \geq a(x)+(b(x) / \rho) e^{-\gamma t} \quad(\rho>0) .
$$

This condition is fulfilled by any nonnegative constant $\gamma$ such that $\gamma \geq a(x)+b(x) / \rho$. The existence of a solution $u(t, x)$ and the relation (2.9) follows from Theorem 2.1. 
When $a(x)<0$ and $b(x) \equiv 0$ in $\bar{\Omega}$, the constant $\gamma$ can be chosen negative. In this situation, the solution $u(t, x)$ decays to zero as $t \rightarrow \infty$. This proves the theorem.

The linear growth condition (2.8) for the global existence of a solution is by no means trivial. It is well known that if $K(x, y) \equiv 0$ and $f(x, u)=a u^{1+\delta}$ for some positive constants $a, \delta$, then the solution of problem (1.1) is positive and blows up in finite time when $u_{0}(x)$ is suitably large (e.g., see $\left.[9,12]\right)$. The magnitude of $u_{0}$ depends on $a, \delta$, and the type of boundary condition, including the size of the domain $\Omega$. Since every nonnegative solution of $(1.1)$ with $K(x, y) \equiv 0$ is a lower solution of the same problem with $K(x, y) \geq 0$ we conclude from Theorem 2.1 that the solution must also blow up in finite time.

3. The steady-state problem. The method of upper and lower solutions for the time-dependent problem (1.1) can be applied to the steady-state problem (1.2) in a similar fashion. The definition of upper and lower solutions for (1.2) is again based on the hypothesis $K(x, y) \geq 0$.

Definition 3.1. A function $\tilde{u}_{s} \in C^{2}(\Omega) \cap C(\bar{\Omega})$ is called an upper solution of (1.2) if

$$
\begin{array}{ll}
-L \tilde{u}_{s} \geq f\left(x, \tilde{u}_{s}\right) & \text { in } \Omega, \\
B\left[\tilde{u}_{s}\right] \geq \int_{\Omega} K(x, y) \tilde{u}_{s}(y) d y & \text { on } \partial \Omega .
\end{array}
$$

Similarly, $\hat{u}_{s}$ is called a lower solution if it satisfies the reversed inequalities in (3.1).

The pair $\tilde{u}_{s}$ and $\hat{u}_{s}$ are said to be ordered if $\tilde{u}_{s} \geq \hat{u}_{s}$ in $\bar{\Omega}$. It is easy to see that $\tilde{u}_{s}$ and $\hat{u}_{s}$ are also upper and lower solutions of (1.1) when $u_{0} \leq \tilde{u}_{s}$ and $u_{0} \geq \hat{u}_{s}$, respectively. Moreover, every (classical) solution of (1.2) is an upper solution as well as a lower solution.

For any pair of ordered upper and lower solutions $\tilde{u}_{s}, \hat{u}_{s}$, we set

$$
\begin{gathered}
\left\langle\hat{u}_{s}, \tilde{u}_{s}\right\rangle \equiv\left\{u \in C(\bar{\Omega}) ; \hat{u}_{s} \leq u \leq \tilde{u}_{s}\right\}, \\
c(x) \equiv \max \left\{-\frac{\partial f}{\partial u}(x, u) ; \hat{u}_{s} \leq u \leq \tilde{u}_{s}\right\}
\end{gathered}
$$

and construct a sequence $\left\{u^{(k)}\right\}$ from the linear iteration process:

$$
\begin{aligned}
-L u^{(k)}+c u^{(k)} & =c u^{(k-1)}+f\left(x, u^{(k-1)}\right) & & \text { in } \Omega, \\
B\left[u^{(k)}\right] & =\int_{\Omega} K(x, y) u^{(k-1)}(y) d y & & \text { on } \partial \Omega,
\end{aligned}
$$

where the initial iteration $u^{(0)}$ is either $\tilde{u}_{s}$ or $\hat{u}_{s}$. Denote the sequence by $\left\{\bar{u}^{(k)}\right\}$ when $u^{(0)}=\tilde{u}_{s}$ and by $\left\{\underline{u}^{(k)}\right\}$ when $u^{(0)}=\hat{u}_{s}$. These sequences possess the following monotone convergence property similar to that in Theorem 2.1.

THEOREM 3.1. Let $K(x, y) \geq 0$ and let $\tilde{u}_{s}, \hat{u}_{s}$ be a pair of ordered upper and lower solutions of (1.2). Then the sequence $\left\{\bar{u}^{(k)}\right\}$ converges monotonically from above to a solution $\bar{u}_{s}$ of (1.2) and the sequence $\left\{\underline{u}^{(k)}\right\}$ converges monotonically from below 
to a solution $\underline{u}_{s}$. Moreover, $\bar{u}_{s} \geq \underline{u}_{s}$ and if $u_{s}^{*}$ is any other solution in $\left\langle\hat{u}_{s}, \tilde{u}_{s}\right\rangle$ then $\bar{u}_{s} \geq u_{s}^{*} \geq \underline{u}_{s}$ in $\bar{\Omega}$.

Proof. It is easily seen by the same argument as in the proof of Theorem 2.1 that the sequence $\left\{\bar{u}^{(k)}\right\}$ is monotone nonincreasing, $\left\{\underline{u}^{(k)}\right\}$ is monotone nondecreasing, and $\bar{u}^{(k)} \geq \underline{u}^{(k)}$ for every $k=1,2, \ldots$. Hence these sequences converge, respectively, to some limits $\bar{u}_{s}$ and $\underline{u}_{s}$, and satisfy the relation $\bar{u}_{s} \geq \underline{u}_{s}$. A regularity argument as in $[9,14]$ shows that $\bar{u}_{s}$ and $\underline{u}_{s}$ are solutions of (1.2). By considering $\left(\tilde{u}_{s}, u_{s}^{*}\right)$ and $\left(u_{s}^{*}, \hat{u}_{s}\right)$ as two pairs of ordered upper and lower solutions of (1.2), the above conclusion implies that $\bar{u}_{s} \geq u_{s}^{*} \geq \underline{u}_{s}$. More detailed arguments can be found in $[9,14]$.

In view of the property $\bar{u}_{s} \geq u_{s}^{*} \geq \underline{u}_{s}$ for any solution $u_{s}^{*}$ in $\left\langle\hat{u}_{s}, \tilde{u}_{s}\right\rangle, \bar{u}_{s}$ and $\underline{u}_{s}$ are often referred to as the maximal and minimal solutions of (1.2), respectively. To guarantee the existence of maximal and minimal solutions we need to find a pair of ordered upper and lower solutions. Such a pair is given by $\tilde{u}_{s}=M$ and $\hat{u}_{s}=m$ when conditions (2.5) and (2.6) are satisfied. As a consequence of Theorem 3.1 we have the following existence-comparison result.

THEOREM 3.2. Let conditions (2.5), (2.6) hold for some constants $M>0, m \leq 0$. Then problem (1.2) has a maximal solution $\bar{u}_{s}$ and a minimal solution $\underline{u}_{s}$ such that

$$
m \leq \underline{u}_{s}(x) \leq \bar{u}_{s}(x) \leq M \quad(x \in \bar{\Omega}) .
$$

Moreover, if $u_{s}^{*}$ is any solution between $m$ and $M$ then $\underline{u}_{s} \leq u_{s}^{*} \leq \bar{u}_{s}$ in $\bar{\Omega}$.

Theorem 3.2 implies that problem (1.2) may have more than one solution between $m$ and $M$. To ensure the uniqueness of the solution we assume that $K(x, y)$ satisfies either the condition

$$
K(x, y) \geq 0, \quad \int_{\Omega} K(x, y) d y<1 \quad(x \in \partial \Omega, y \in \Omega)
$$

or the condition

$$
K(x, y)>0, \quad \int_{\Omega} K(x, y) d y \leq 1, \quad \int_{\Omega} K(x, y) d y \not \equiv 1 \quad(x \in \partial \Omega, y \in \Omega) .
$$

The following lemma is crucial for the uniqueness property of a solution:

Lemma 3.1. Let $K(x, y)$ satisfy either (3.5) or (3.6), and let $w \in C^{2}(\Omega) \cap C(\bar{\Omega})$ be such that

$$
\begin{array}{lll}
-L w \leq 0 & & \text { in } \Omega, \\
B[w] \leq \int_{\Omega} K(x, y) w(y) d y & & \text { on } \partial \Omega .
\end{array}
$$

Then $w(x) \leq 0$ in $\bar{\Omega}$.

Proof. Assume, by contradiction, that $w(x)$ is not nonpositive in $\bar{\Omega}$. Then there exists $x_{0} \in \bar{\Omega}$ such that $w\left(x_{0}\right)$ is a positive maximum. By the maximum principle, $x_{0} \notin \Omega$ unless $w(x)$ is a positive constant (cf. [13]). It is easily seen from the boundary condition in (3.7) that $w(x)$ can be a positive constant only when

$$
\int K(x, y) d y \geq 1 \text { for all } x \in \partial \Omega,
$$


which contradicts both conditions (3.5) and (3.6). Hence $x_{0} \in \partial \Omega$ and $w(x)$ is not a constant. By the strong maximum principle, $\partial w / \partial \nu>0$ at $x_{0}$. Since by (3.7) and the definition of $B[w]$,

$$
\alpha_{0}(\partial w / \partial \nu)\left(x_{0}\right)+w\left(x_{0}\right) \leq\left(\int_{\Omega} K\left(x_{0}, y\right) d y\right) w\left(x_{0}\right)
$$

we see that this is not possible when either condition (3.5) holds or $\alpha_{0}>0$ and condition (3.6) holds. In the case $\alpha_{0}=0$, the boundary condition at $x_{0}$ is reduced to

$$
w\left(x_{0}\right) \leq \int_{\Omega} K\left(x_{0}, y\right) w(y) d y .
$$

Since, by hypothesis, $K\left(x_{0}, y\right)>0$ in $\Omega$ and $w(x)$ is a nonconstant nonnegative continuous function in $\Omega$, the above relation implies that

$$
w\left(x_{0}\right)<\left(\int_{\Omega} K\left(x_{0}, y\right) d y\right) w\left(x_{0}\right) \text {. }
$$

This again leads to a contradiction which yields the result $w(x) \leq 0$ in $\bar{\Omega}$.

As a direct consequence of Lemma 3.1 we have the following uniqueness result when $f(x, u)$ is nonincreasing in $u$.

THEOREM 3.3. Let $\tilde{u}_{s}, \hat{u}_{s}$ be a pair of ordered upper and lower solutions of (1.2), and let $K(x, y)$ satisfy either (3.5) or (3.6). If

$$
f_{u}(x, u) \leq 0 \text { for } u \in\left\langle\hat{u}_{s}, \tilde{u}_{s}\right\rangle \quad(x \in \Omega)
$$

then problem (1.2) has a unique solution $u_{s}$ such that $\hat{u}_{s} \leq u_{s} \leq \tilde{u}_{s}$.

Proof. By Theorem 3.1, Problem 2.1 has a maximal solution $\bar{u}_{s}$ and a minimal solution $\underline{u}_{s}$ and $\bar{u}_{s} \geq \underline{u}_{s}$. Hence to prove the uniqueness of the solution $u_{s}$ it suffices to show $\bar{u}_{s}=\underline{u}_{s}$. Let $w=\bar{u}_{s}-\underline{u}_{s}$. By (1.2), (3.8), and $\bar{u}_{s} \geq \underline{u}_{s}$

$$
\begin{aligned}
& -L w=f\left(x, \bar{u}_{s}\right)-f\left(x, \underline{u}_{s}\right) \leq 0 \text { in } \Omega, \\
& B[w]=\int_{\Omega} K(x, y) w(y) d y \quad \text { on } \partial \Omega .
\end{aligned}
$$

It follows from Lemma 3.1 that $w(x) \leq 0$, or equivalently, $\bar{u}_{s} \leq \underline{u}_{s}$. This leads to the conclusion $\bar{u}_{s}=\underline{u}_{s}$.

When $f(x, u)$ satisfies condition (2.6), $\tilde{u}_{s}=M$ and $\hat{u}_{s}=m$ are ordered upper and lower solutions of (1.2). By an application of Theorem 3.3 we have the following conclusion.

Corollary 3.1. Let $K(x, y)$ satisfy either (3.5) or (3.6) and let there exist constants $M>0, m \leq 0$ such that

$$
f(x, M) \leq 0, \quad f(x, m) \geq 0, \quad f_{u}(x, u) \leq 0 \text { for } m \leq u \leq M .
$$

Then problem (1.2) has a unique solution $u_{s}(x)$ such that $m \leq u_{s}(x) \leq M$. If $f(x, 0) \geq 0$ and $f(x, 0) \not \equiv 0$ in $\Omega$, then $u_{s}(x)>0$ in $\Omega$ when $\alpha_{0}=0$, and $u_{s}(x)>0$ in $\bar{\Omega}$ when either $\alpha_{0}>0$ or (3.6) holds.

Proof. The existence and uniqueness of a solution $u_{s}$ follows from Theorem 3.3 with $\tilde{u}_{s}=M$ and $\hat{u}_{s}=m$. To show the positive property of $u_{s}$ we observe that 
$\hat{u}_{s}=0$ is a lower solution and is not a true solution when $f(x, 0) \geq 0$ and $f(x, 0) \not \equiv$ 0 . Using $m=0$ in (3.9) leads to $u_{s} \geq 0$. Since by the mean-value theorem,

$$
-L u_{s}=f\left(x, u_{s}\right)=\left(f_{u}(x, \eta)\right) u_{s}+f(x, 0)
$$

for some $\eta \in\left[0, u_{s}\right]$, the hypothesis $f(x, 0) \geq 0$ ensures that

$$
L u_{s}+c^{*}(x) u_{s} \leq 0 \quad \text { in } \Omega
$$

where $c^{*}(x) \equiv f_{u}(x, \eta(x)) \leq 0$. Now if $u_{s}\left(x_{0}\right)=0$ at some $x_{0} \in \bar{\Omega}$, then the maximum principle implies that $x_{0}$ cannot be in $\Omega$ unless $u_{s}$ is a constant. Since $u_{s} \geq 0$ and $u_{s} \equiv 0$ is not a solution we conclude that either $u_{s}$ is a positive constant or $u_{s}>0$ in $\Omega$. However, by (3.5) or (3.6), since $u_{s}$ cannot be a constant it follows that $u_{s}>0$ in $\Omega$. Moreover, if $u_{s}\left(x_{0}\right)=0$ at some $x_{0} \in \partial \Omega$ then $\partial u_{s} / \partial \nu<0$ at $x_{0}$ and

$$
\alpha_{0}\left(\partial u_{s} / \partial \nu\right)\left(x_{0}\right)=\int_{\Omega} K\left(x_{0}, y\right) u_{s}(y) d y .
$$

The above relation cannot hold when either $\alpha_{0}>0$ or (3.6) holds. This proves $u_{s}(x)>0$ in $\bar{\Omega}$.

It should be pointed out that the last condition in (3.6) for the uniqueness result cannot be removed without additional requirements on $f(x, u)$. For example, the trivial function $f(x, u) \equiv 0$ satisfies condition (3.9), and if $\int_{\Omega} K(x, y) d y \equiv 1$ then every constant is a solution of (1.2).

4. Asymptotic behavior. In this section we investigate the asymptotic behavior of the time-dependent solution of (1.1) in relation to a steady-state solution of (1.2). In the absence of an explicit knowledge of the steady-state solution which may be nonuniform in $\Omega$, we establish a monotone property of the time-dependent solution when the initial function $u_{0}(x)$ is either an upper solution or a lower solution of (1.2). This property is based on the following analogous result to that in Lemma 3.1.

LEMMA 4.1. Let $K(x, y)$ satisfy either (3.5) or (3.6), and let $w \in C^{1,2}\left(D_{T}\right) \cap C\left(\bar{D}_{T}\right)$ be such that

$$
\begin{array}{ll}
w_{t}-L w+c w \leq 0 & \text { in } D_{T}, \\
B[w] \leq \int_{\Omega} K(x, y) w(t, y) d y & \text { on } S_{T}, \\
w(0, x) \leq 0 & \text { in } \Omega,
\end{array}
$$

where $c \equiv c(t, x)$ is a bounded function in $D_{T}$. Then $w(t, x) \leq 0$ in $\bar{D}_{T}$.

Proof. By replacing $w$ by the function $e^{-\gamma t} w$ for some constant $\gamma$ satisfying $\gamma \geq-c(t, x)$ if necessary, we may assume that $c(t, x) \geq 0$ in $D_{T}$. Assume by contradiction that $w$ is not nonpositive in $\bar{D}_{T}$. Then there exists a point $\left(t_{0}, x_{0}\right) \in$ $\bar{D}_{T}$ such that $w\left(t_{0}, x_{0}\right)$ is a positive maximum. By the relation $w(0, x) \leq 0$, $t_{0}>0$. Since $c(t, x) \geq 0$, the maximum principle implies that $x_{0} \notin \Omega$ unless $w$ is a positive constant in $\bar{D}_{T}$. However, by the boundary inequality in (4.1) and the condition (3.5) or (3.6), $w$ cannot be a positive constant, and so we must have $x_{0} \in \partial \Omega$. It follows from $K(x, y) \geq 0$ and the positive maximum property of 
$w\left(t_{0}, x_{0}\right)$ that

$$
\alpha_{0}(\partial w / \partial \nu)\left(t_{0}, x_{0}\right)+w\left(t_{0}, x_{0}\right) \leq\left(\int_{\Omega} K\left(x_{0}, y\right) d y\right) w\left(t_{0}, x_{0}\right) .
$$

Since, by the strong maximum principle, $(\partial w / \partial \nu)\left(t_{0}, x_{0}\right)>0$, the above inequality cannot be satisfied when either condition (3.5) holds or $\alpha_{0}>0$ and condition (3.6) holds. In the case $\alpha_{0}=0$, the boundary inequality at $\left(t_{0}, x_{0}\right)$ is reduced to

$$
w\left(t_{0}, x_{0}\right) \leq \int_{\Omega} K\left(x_{0}, y\right) w\left(t_{0}, y\right) d y .
$$

Since $w$ is not a constant, condition (3.6) leads again to a contradiction. This shows that $w$ must be nonpositive in $\bar{D}_{T}$.

A direct consequence of Lemma 4.1 is the monotone property of the time-dependent solution of (1.1) when the initial function $u_{0}$ is either an upper solution or a lower solution of the steady-state problem (1.2).

LEMMA 4.2. Let $\tilde{u}_{s}, \hat{u}_{s}$ be a pair of ordered upper and lower solutions of (1.2), and let $\bar{u}(t, x), \underline{u}(t, x)$ be the solutions of $(1.1)$ with $\bar{u}(0, x)=\tilde{u}_{s}(x)$ and $\underline{u}(0, x)=$ $\hat{u}_{s}(x)$, respectively. Assume that either (3.5) or (3.6) holds. Then for each $x \in \bar{\Omega}$, $\bar{u}(t, x)$ is monotone nonincreasing and $\underline{u}(t, x)$ is monotone nondecreasing in $t$ for $t>0$. Moreover,

$$
\bar{u}(t, x) \geq \underline{u}(t, x) \text { for all } t \geq 0, x \in \bar{\Omega} .
$$

Proof. Since $\tilde{u}_{s}, \hat{u}_{s}$ are also ordered upper and lower solutions of (1.1) when $\tilde{u}_{s} \geq u_{0} \geq \hat{u}_{s}$, Theorem 2.1 ensures that the solutions $\bar{u}(t, x)$ and $\underline{u}(t, x)$ exist and satisfy the relation

$$
\hat{u}_{s}(x) \leq \bar{u}(t, x) \leq \tilde{u}_{s}(x), \quad \hat{u}_{s}(x) \leq \underline{u}(t, x) \leq \tilde{u}_{s}(x) .
$$

Consider the function $w(t, x) \equiv \bar{u}(t+\delta, x)-\bar{u}(t, x)$ for an arbitrary constant $\delta>0$. In view of (1.1) and the mean-value theorem,

$$
\begin{aligned}
w_{t}-L w & =f(x, \bar{u}(t+\delta))-f(x, \bar{u}(t, x))=\left(f_{u}(x, \eta)\right) w, \\
B[w] & =\int_{\Omega} K(x, y) w(t, y) d y, \\
w(0, x) & =\bar{u}(\delta, x)-\bar{u}(0, x)=\bar{u}(\delta, x)-\tilde{u}_{s}(x),
\end{aligned}
$$

where $\eta \equiv \eta(t, x)$ is an intermediate value between $\bar{u}(t+\delta, x)$ and $\bar{u}(t, x)$. Since $f_{u}(x, \eta)$ is bounded in $D_{T}$ for every $T>0$ and, by $(4.3), w(0, x) \leq 0$, Lemma 4.1 implies that $w(t, x) \leq 0$ in $\bar{D}_{T}$. This leads to $\bar{u}(t+\delta, x) \leq \bar{u}(t, x)$ for every $t>0$. The arbitrariness of $\delta>0$ ensures that $\bar{u}(t, x)$ is nonincreasing in $t$. A similar argument shows that $\underline{u}(t, x)$ is nondecreasing in $t$ for every $t>0$. To show the relation $\bar{u}(t, x) \geq \underline{u}(t, x)$ we observe that the function $w^{*}(t, x) \equiv \underline{u}(t, x)-\bar{u}(t, x)$ satisfies the relation (4.4) with $w^{*}(0, x)=\hat{u}_{s}(x)-\tilde{u}_{s}(x)$ and with possibly a different intermediate value $\eta(t, x)$. Since $w^{*}(0, x) \leq 0$, the same reasoning as for $w(t, x)$ shows that $w^{*}(t, x) \leq 0$ in $\bar{D}_{T}$ for every $T>0$. This gives the relation (4.2).

When the initial function $u_{0}$ is in $\left\langle\hat{u}_{s}, \tilde{u}_{s}\right\rangle$ but is not necessarily an upper solution nor a lower solution the same argument as in the proof of Lemma 4.2 leads to the following comparison result. 
Lemma 4.3. Let $u(t, x)$ be the solution of $(1.1)$ corresponding to any $u_{0} \in\left\langle\hat{u}_{s}, \tilde{u}_{s}\right\rangle$, and let either (3.5) or (3.6) hold. Then

$$
\underline{u}(t, x) \leq u(t, x) \leq \bar{u}(t, x) \text { for all } t>0, x \in \bar{\Omega} .
$$

Proof. Let $w(t, x)=u(t, x)-\bar{u}(t, x)$. Then $w(t, x)$ satisfies $(4.4)$ with $w(0, x)$ $=u_{0}(x)-\tilde{u}_{s}(x) \leq 0$ and with $\eta(t, x)$ an intermediate value between $u(t, x)$ and $\bar{u}(t, x)$. By an application of Lemma 4.1, w(t,x) $\leq 0$ which gives $u(t, x) \leq$ $\bar{u}(t, x)$. The proof for $u(t, x) \geq \underline{u}(t, x)$ is similar.

Based on Lemmas 4.2 and 4.3 we have the following monotone convergence of the time-dependent solutions $\bar{u}(t, x)$ and $\underline{u}(t, x)$.

THEOREM 4.1. Let $\tilde{u}_{s}, \hat{u}_{s}$ be a pair of ordered upper and lower solutions of (1.2), and let $\bar{u}(t, x), \underline{u}(t, x)$ be the solutions of $(1.1)$ with $\bar{u}(0, x)=\tilde{u}_{s}(x)$ and $\underline{u}(0, x)=$ $\hat{u}_{s}(x)$, respectively. Assume that either condition (3.5) or condition (3.6) holds. Then

$$
\lim \bar{u}(t, x)=\bar{u}_{s}(x), \quad \lim \underline{u}(t, x)=\underline{u}_{s}(x) \quad \text { as } t \rightarrow \infty,
$$

where $\bar{u}_{s}(x)$ and $\underline{u}_{s}(x)$ are the maximal and minimal solutions of (1.2), respectively.

Proof. By Lemma 4.2 and the relations (4.2) and (4.3), the limits

$$
\lim \bar{u}(t, x)=\bar{u}_{s}^{*}(x), \quad \lim \underline{u}(t, x)=\underline{u}_{s}^{*}(x) \quad \text { as } t \rightarrow \infty \quad(x \in \bar{\Omega})
$$

exist and satisfy $\tilde{u}_{s} \geq \bar{u}_{s}^{*} \geq \underline{u}_{s}^{*} \geq \hat{u}_{s}$ in $\bar{\Omega}$. It can be shown by a similar argument as in [9] that $\bar{u}_{s}^{*}$ and $\underline{u}_{s}^{*}$ are solutions of (1.2). To complete the proof of the theorem it suffices to show that $\bar{u}_{s}^{*}=\bar{u}_{s}$ and $\underline{u}_{s}^{*}=\underline{u}_{s}$.

Consider the maximal solution $\bar{u}_{s}$ as a solution of (1.1) with $u_{0}=\bar{u}_{s}$. By Lemma 4.3, $\underline{u}(t, x) \leq \bar{u}_{s}(x) \leq \bar{u}(t, x)$. Letting $t \rightarrow \infty$ and using the relation (4.7) yields $\underline{u}_{s}^{*} \leq \bar{u}_{s} \leq \bar{u}_{s}^{*}$. Since $\bar{u}_{s}^{*} \in\left\langle\hat{u}_{s}, \tilde{u}_{s}\right\rangle$ and $\bar{u}_{s}$ is the maximal solution in $\left\langle\hat{u}_{s}, \tilde{u}_{s}\right\rangle$ we must have $\bar{u}_{s}^{*} \leq \bar{u}_{s}$. This leads to $\bar{u}_{s}^{*}=\bar{u}_{s}$. The proof for $\underline{u}_{s}^{*}=\underline{u}_{s}$ is similar.

Theorem 4.1 gives the monotone convergence of the time-dependent solution to a steady-state solution when the initial function is either an upper solution or a lower solution of the steady-state problem. For an arbitrary initial function in $\left\langle\hat{u}_{s}, \tilde{u}_{s}\right\rangle$ we have the following conclusion.

THEOREM 4.2. Let $\tilde{u}_{s}, \hat{u}_{s}$ be a pair of ordered upper and lower solutions of (1.2), and let either condition (3.5) or condition (3.6) hold. Then for any $u_{0} \in\left\langle\hat{u}_{s}, \tilde{u}_{s}\right\rangle$ the solution $u(t, x)$ of $(1.1)$ converges to a solution $u_{s}(x)$ of (1.2) as $t \rightarrow \infty$ if and only if $u_{s}(x)$ is unique in $\left\langle\hat{u}_{s}, \tilde{u}_{s}\right\rangle$.

Proof. Suppose $u_{s}(x)$ is the unique solution of $(1.2)$ in $\left\langle\hat{u}_{s}, \tilde{u}_{s}\right\rangle$. Then $\bar{u}_{s}=\underline{u}_{s}=$ $u_{s}$ and

$$
\lim \bar{u}(t, x)=\lim \underline{u}(t, x)=u_{s}(x) \quad \text { as } t \rightarrow \infty \quad(x \in \bar{\Omega}) .
$$

Since by Lemma $4.3, u(t, x)$ satisfies relation (4.5) it follows that $u(t, x)$ must also converge to $u_{s}(x)$ as $t \rightarrow \infty$. Conversely, if $u_{s}^{*}$ is a solution of (1.2) in $\left\langle\hat{u}_{s}, \tilde{u}_{s}\right\rangle$ then it is also a solution of (1.1) with $u_{0}=u_{s}^{*}$. Since $u_{s}^{*}$ is independent of $t$, it converges to $u_{s}$ only when $u_{s}^{*}=u_{s}$. This shows that $u_{s}$ is unique in $\left\langle\hat{u}_{s}, \tilde{u}_{s}\right\rangle$.

A direct consequence of Theorem 4.2 yields the following dynamical behavior of the problem (1.1). 
TheOREM 4.3. Let $K(x, y)$ satisfy either (3.5) or (3.6), and let $f(x, u)$ satisfy (3.9) for some $M>0, m \leq 0$. Then for any $u_{0}(x)$ with $m \leq u_{0}(x) \leq M$ the solution $u(t, x)$ of (1.1) converges to a unique solution $u_{s}(x)$ of $(1.2)$ as $t \rightarrow \infty$.

Proof. By condition (3.9), $\tilde{u}_{s}=M$ and $\hat{u}_{s}=m$ are ordered upper and lower solutions of (1.2). Since by Corollary 3.1, problem (1.2) has a unique solution $u_{s}$ in $\left\langle\hat{u}_{s}, \tilde{u}_{s}\right\rangle$, the conclusion of the theorem follows from Theorem 4.2.

In the special case $f(x, u)=c(x) u$ where $c(x) \leq 0$, condition (3.9) is satisfied by any constants $M>0$ and $m \leq 0$. Hence if $K(x, y)$ satisfies either (3.5) or (3.6) then by Corollary 3.1, $u_{s}=0$ is the only solution of (1.2). The arbitrariness of $M>0, m \leq 0$, and Theorem 4.3 imply that for any initial function $u_{0}$ the corresponding solution $u(t, x)$ of (1.1) converges to 0 as $t \rightarrow \infty$. This shows that the trivial solution $u_{s}=0$ is globally asymptotically stable. However, if $K(x, y)$ is not always nonnegative as in the case considered in [3, 4, 7], Theorem 4.3 is not directly applicable. To treat this case we use solutions of $(1.1)$ with $K(x, y) \geq 0$ as comparison functions for the solution with a general $K(x, y)$.

Consider the time-dependent problem

$$
\begin{array}{ll}
U_{t}-L U=f(x, U) & (t>0, x \in \Omega), \\
B[U]=\int_{\Omega}|K(x, y)| U(t, y) d y & (t>0, x \in \partial \Omega), \\
U(0, x)=U_{0}(x) & (x \in \Omega),
\end{array}
$$

where $U_{0} \geq 0$. Assume that $|K(x, y)|$ satisfies (2.5) and $f(x, u)$ satisfies (2.6) with $m=0$. By Theorem 2.2, problem (4.8) has a unique nonnegative solution $U$ in $\langle 0, M\rangle$ whenever $U_{0} \leq M$. Upon replacing $U, U_{0}$ by $-U$ and $-U_{0}$, respectively, in (4.8), the resulting problem has a unique nonpositive solution $U^{*}$ in $\left\langle-M^{*}, 0\right\rangle$ provided that $f(x, 0) \leq 0$ and $f\left(x,-M^{*}\right) \geq 0$ for some constant $M^{*} \geq U_{0}$. This follows from the fact that $\tilde{u}=0$ and $\hat{u}=-M^{*}$ are ordered upper and lower solutions. It is easy to see that $U^{*}=-U$ if

$$
M^{*}=M \text { and } f(x,-u)=-f(x, u) \text { for }|u| \leq M .
$$

Using $U$ and $-U$ as comparison functions we have the following comparison result for problem (1.1) where $K(x, y)$ may assume positive as well as negative values on $\partial \Omega \times \Omega$.

THEOREM 4.4. Let $|K(x, y)|$ satisfy either condition (3.5) or condition (3.6), and let there exist a constant $M>0$ such that

$$
f(x, M) \leq 0, f(x, u)=-f(x,-u) \text { for }|u| \leq M .
$$

Denote by $u(t, x), U(t, x)$ the respective solutions of (1.1) and (4.8), where $U_{0}=$ $\left|u_{0}\right| \leq M$. Then

$$
-U(t, x) \leq u(t, x) \leq U(t, x) \quad(t>0, x \in \bar{\Omega}) .
$$

Proof. Let $w=e^{\gamma t}(u-U)$, where $\gamma$ is any constant satisfying the relation $\gamma \geq$ $f_{u}(x, u)$ for $x \in \bar{\Omega},|u| \leq M$. By (1.1), (4.8), and the mean-value theorem, $w$ 
satisfies

$$
\begin{aligned}
w_{t}-L w+\gamma w=e^{-\gamma t}[f(x, u)-f(x, U)]=\left(f_{u}(x, \eta)\right) w, \\
B[w]=e^{-\gamma t} \int_{\Omega}[K(x, y) u(t, y)-|K(x, y)| U(t, y)] d y, \\
\quad=\int_{\Omega} K(x, y) w(t, y) d y+e^{-\gamma t} \int_{\Omega}[K(x, y)-|K(x, y)|] U(t, y) d y .
\end{aligned}
$$

Since $U(t, x) \geq 0$ the above relation is reduced to

$$
\begin{aligned}
& w_{t}-L w+c^{*}(t, x) w=0 \quad(t>0, x \in \Omega), \\
& B[w] \leq \int_{\Omega} K(x, y) w(t, y) d y \quad(t>0, x \in \partial \Omega),
\end{aligned}
$$

where $c^{*}(t, x)=\gamma-f_{u}(x, \eta) \geq 0$. We show that $w(t, x) \leq 0$. Assume this is not true. Then there exists a point $\left(t_{0}, x_{0}\right)$ such that $w\left(t_{0}, x_{0}\right)$ is a positive maximum. Since $w(0, x)=U_{0}-\left|U_{0}\right| \leq 0, t_{0} \neq 0$. By the maximum principle and condition (3.5) or (3.6), $x_{0} \in \partial \Omega$ and $w$ is not a constant. In view of the boundary inequality in $(4.11)$,

$$
B\left[w\left(t_{0}, x_{0}\right)\right] \leq\left(\int_{\Omega}\left|K\left(x_{0}, y\right)\right| d y\right) w\left(t_{0}, x_{0}\right) .
$$

It follows from the same argument as in the proof of Lemma 4.1 that the above inequality cannot be true. This contradiction shows that $w(t, x) \leq 0$ which gives the result $u \leq U$. Since by (4.9), $-U$ is a solution of (4.8) with $U_{0}$ replaced by $-U_{0}$, a similar argument as above shows that $u \geq-U$. This proves the theorem.

Based on the comparison result in Theorem 4.4 we have the following decay property of the time-dependent solution.

TheOREM 4.5. Let $|K(x, y)|$ satisfy either condition (3.5) or condition (3.6), and let $f(x, u)$ satisfy condition (4.9) and the nonincreasing property $f_{u}(x, u) \leq 0$ for $0 \leq u \leq M$. Then for any $u_{0}$ with $\left|u_{0}\right| \leq M$ the corresponding solution $u(t, x)$ of (1.1) possesses the decay property

$$
\lim |u(t, x)|=0 \quad \text { as } t \rightarrow \infty \quad(x \in \bar{\Omega}) .
$$

Proof. Since by (4.9) $f(x, 0)=0$, Corollary 3.1 implies that $u_{s}=0$ is the unique solution of (1.2) in $\langle 0, M\rangle$. Hence by Theorem 4.3 the solution $U(t, x)$ of $(4.8)$ with $U_{0}=\left|u_{0}\right|$ converges to 0 as $t \rightarrow \infty$. The decay property of $|u(t, x)|$ follows from Theorem 4.4.

When the function $f(x, u)$ is given in the form

$$
f(x, u)=c(x) u^{2 p+1}, \quad p=0,1,2, \ldots,
$$

where $c(x) \leq 0$, the requirements on $f$ in Theorem 4.5 are fulfilled by any constant $M>0$. Hence given any initial function $u_{0}$ the solution $u(t, x)$ of (1.1) decays to 0 as $t \rightarrow \infty$ when $|K(x, y)|$ satisfies either condition (3.5) or condition (3.6). This decay property was also obtained by Day [4] and Friedman [7] for the linear case $f(x, u)=c(x) u$ where $|K(x, y)|$ is required to satisfy condition (3.5). 


\section{REFERENCES}

[1] J. W. Bebernes and R. Ely, Comparison techniques and the method of lines for a parabolic functional equation, Rocky Mountain J. Math. 12, 723-733 (1982)

[2] V. Capasso and $\mathrm{K}$. Kunisch, $A$ reaction-diffusion system arising in modelling man-environment diseases, Quart. Appl. Math. 46, 431-450 (1988)

[3] W. A. Day, Extensions of a property of the heat equation to linear thermoelasticity and other theories, Quart. Appl. Math. 40, 319-330 (1982)

[4] W. A. Day, A decreasing property of solutions of parabolic equations with applications to thermoelasticity, Quart. Appl. Math. 40, 468-475 (1983)

[5] P. De Mottoni, E. Orlandi, and A. Tesei, Asymptotic behavior for a system describing epidemics with migration and spatial spread of infection, J. Nonlinear Analysis 3, 663-675 (1979)

[6] K. Deng, Comparison principle for some nonlocal problems, Quart. Appl. Math. 50, 517-522 (1992)

[7] A. Friedman, Monotonic decay of solutions of parabolic equations with nonlocal boundary conditions, Quart. Appl. Math. 44, 401-407 (1986)

[8] B. Kawohl, Remarks on a paper by $\boldsymbol{W}$. A. Day on a maximum principle under nonlocal boundary conditions, Quart. Appl. Math. 44, 751-752 (1987)

[9] C. V. Pao, Nonlinear Parabolic and Elliptic Equations, Plenum Press, New York, 1992

[10] C. V. Pao, Blowing-up of solution for a nonlocal reaction-diffusion problem in combustion theory, J. Math. Anal. Appl. 166, 591-600 (1992)

[11] C. V. Pao, On nonlinear reaction-diffusion systems, J. Math. Anal. Appl. 87, 165-198 (1982)

[12] C. V. Pao, Asymptotic stability and nonexistence of global solutions for a semilinear parabolic equation, Pacific J. Math. 84, 191-197 (1979)

[13] M. H. Protter and H. F. Weinberger, Maximum Principles in Differential Equations, Prentice-Hall, Englewood Cliffs, NJ, 1967

[14] D. H. Sattinger, Monotone methods in nonlinear elliptic and parabolic boundary-value problems, Indiana Univ. Math. J. 21, 979-1000 (1972) 